\title{
Development of nutritional studies in China
}

\author{
Shankuan Zhu (iD) ${ }^{1}$
}

Received: 2 July 2020 / Revised: 2 October 2020 / Accepted: 26 October 2020 / Published online: 7 November 2020

(c) Springer Nature Limited 2020

Driven by rapid economic growth and drastic shifts in diets, China has experienced an increased burden of nutritionrelated diseases. Take the commonly reported diseases-like type 2 diabetes mellitus, cardiovascular disease, and hypertension for example, their prevalence increased approximately $4-12$-folds in the past decades and account for $70 \%$ of disability-adjusted life-years lost and $80 \%$ of deaths in China [1-4]. Along with these changes, the focus of nutritional researches has transformed from food security and availability to better nutrition and health. Consider the significant growth in nutritional researches that investigate dietary transitions and diseases burdens; or nutritional risks in vulnerable populations; or changes in dietary intakes and food environment between urban and rural areas; or the development of nutrition policies, the aim of this special issue is to include representative studies that provide a timely discussion on important topics in nutrition, especially those with direct translation to public health and disease prevention.

Due to urbanization, nutrition transition in China has been substantial. Dietary pattern has undergone rapid westernization that changes the traditional dietary pattern, characterized as high carbohydrates and low protein and fat intakes, to the western dietary pattern by increasing consumption of animal proteins, dairy products, refined grains [5], and sugar sweetened beverages [6]. Similar to Western countries, the available evidence indicated that the increased consumption of red meat, processed meat, and sugar sweetened beverage associated with increased cardiometabolic disease burdens in China [7].

Shankuan Zhu

zsk@zju.edu.cn

1 Chronic Disease Research Institute, Department of Nutrition and Food Hygiene, The Children's Hospital, and National Clinical Research Center for Child Health, School of Public Health, School of Medicine, Zhejiang University, 866 Yu-hang-tang Road, Hangzhou, 310058 Zhejiang, China
Although urbanization in the rural areas has improved individuals' living standards, most studies have shown that rural-urban discrepancies of dietary patterns still exist and are associated disease burden [8,9]. All these changes have come along with new health problems, such as overweight and obesity $[10,11]$, which merits more attention on nutrition researches to address the concerns related to dietary transition.

Another important dieting trend that cannot be ignored is the booming online-to-offline (O2O) food delivery industry. While the development of food delivery makes foods so convenient and accessible for people, it has a significant impact on individual's eating behavior. Consumption of take-out foods tends to have higher oil, salt, unhealthy fat, and sugars compared to the home-made foods [12]. In addition, there is also potential food safety risks, which might cause food-borne illness [13]. The current issue reports a review that updates the impact of $\mathrm{O} 2 \mathrm{O}$ food delivery on health outcomes.

Finally, we include discussions on nutrition policies in the current issue to demonstrate the development of policies in China. The government has issued several nutrition policies to achieve specific goals during different times $[14,15]$. Accordingly, the nutrition guidelines for Chinese populations have been updated nearly every 10 years [16]. All these policies and guidelines successfully reduce the prevalence of malnutrition decreases from 6.0 to $2.5 \%$ in China [9]. However, we are confronting a new challenging as the prevalence of obesity increase from 5.6 to $11.9 \%$ [17]. The latest version of nutrition guidelines came out in 2016 with a renewed emphasis on improving food diversity and quality of diet, which calls for fewer sugar intake and highlights the importance of balanced diet [16]. This is not the end. We need to put effect into generating more scientific evidence to specify and improve current nutrition policies and guidelines in order to prevent chronic diseases along with the rising obesity and chronic disease rates. 


\section{Compliance with ethical standards}

Conflict of interest The author declares that he has no conflict of interest.

Publisher's note Springer Nature remains neutral with regard to jurisdictional claims in published maps and institutional affiliations.

\section{References}

1. Yang X, Li J, Hu D, Chen J, Li Y, Huang J, et al. Predicting the 10-year risks of atherosclerotic cardiovascular disease in Chinese population: the China-PAR Project (Prediction for ASCVD Risk in China). Circulation. 2016;134:1430-40.

2. Wang Z, Chen Z, Zhang L, Wang X, Hao G, Zhang Z, et al. Status of hypertension in China: results from the China Hypertension Survey, 2012-2015. Circulation. 2018;137:2344-56.

3. Ma RCW. Epidemiology of diabetes and diabetic complications in China. Diabetologia. 2018;61:1249-60.

4. Tian M, Chen Y, Zhao R, Chen L, Chen X, Feng D, et al. Chronic disease knowledge and its determinants among chronically ill adults in rural areas of Shanxi Province in China: a cross-sectional study. BMC Public Health. 2011;11:948.

5. Ding G, Chen J. Strengthen national nutrition surveillance and adopt effective intervention strategies to reduce the incidence of nutritional diseases. Chin J Epidemiol. 2018;48:517-22.

6. Wang J, Lin X, Bloomgarden ZT, Ning G. The Jiangnan diet, a healthy diet pattern for Chinese. J diabetes. 2020;12:365-71.

7. He Y, Li Y, Yang X, Hemler EC, Fang Y, Zhao L, et al. The dietary transition and its association with cardiometabolic mortality among Chinese adults, 1982-2012: a cross-sectional population-based study. lancet Diabetes Endocrinol. 2019;7: $540-8$.

8. Liu X, Tian Q, Yan D, Cui X. Analysis of dietary structure differences between urban and rural residents in China-Based on the perspective of nutrition level. Acta Nutrimenta Sin. 2016;4:332-6.

9. Zhao L, Guo Q, Li S, Zhao W, Ding G. Strengthening nutrition investigation and monitoring to improve nutrition and health status of Chinese residents. Health Res. 2019;48:517-22.

10. Du SF, Wang HJ, Zhang B, Zhai FY, Popkin BM. China in the period of transition from scarcity and extensive undernutrition to emerging nutrition-related non-communicable diseases, 1949-1992. Obes Rev. 2014;15:8-15.

11. He Y, Ma G, Zhai F, Li Y, Hu Y, Feskens EJ, et al. Dietary patterns and glucose tolerance abnormalities in Chinese adults. Diabetes Care. 2009;32:1972-6.

12. Jaworowska A, Blackham T, Davies IG, Stevenson L. Nutritional challenges and health implications of takeaway and fast food. Nutr Rev. 2013;71:310-8.

13. Gizaw Z. Public health risks related to food safety issues in the food market: a systematic literature review. Environ Health Prev Med. 2019;24:68.

14. Huang $\mathrm{H}$, Zhang B, Du W, Zhang J, Wang H, Liu A. et al. Analysis on nutritional policy and action plan in China. Chin J Health Educ. 2011;27:930-2.

15. Wang Z, Sun J, Wang H, Liu A, Zhang B, Ding G. Dietary structure transition and development of nutrition intervention strategises in China. Acta Nutrimenta Sin. 2019;41:427-32.

16. Liu R, Hong J, Xu X, Feng Q, Zhang D, Gu Y, et al. Gut microbiome and serum metabolome alterations in obesity and after weight-loss intervention. Nat Med. 2017;23:859-68.

17. Gu J. The report on the nutrition and chronic diseases of Chinese residents. Acta Nutrimenta Sin. 2016;38:525-9. 